\title{
MANUEL LACUNZA Y LA VENIDA DEL MESÍAS EN GLORIA Y MAJESTAD: BIBLIOGRAFÍA COMENTADA ${ }^{1}$
}

\author{
Osvaldo Arce \\ Universidad de Chile \\ osvaldarc@yahoo.es
}

Manuel Lacunza y Díaz (1731-1801) es una de las figuras del pensamiento religioso americano que ha provocado más discusión, admiración o rechazo por parte de la crítica a través del tiempo. Así es como teólogos, estudiosos o simples creyentes se han sumado al debate que ha suscitado el libro La Venida del Mesías en gloria y majestad, desde que apareció primero a través de manuscritos parciales (muchos de ellos espurios) a finales del siglo XVIII, y ya en formato de libro en las primeras décadas del XIX (alrededor de 1811). La obra de Lacunza se puede insertar dentro del contexto de las corrientes religiosas llamadas milenaristas (creencias en el reino milenario de Cristo en la tierra), que se manifestaron profusamente en la América Latina de la época colonial.

La discusión crítica sobre La Venida del Mesías en gloria y majestad, a lo largo de los siglos, se puede dividir en tres etapas representativas. La primera podemos situarla en la época en que entran en circulación los manuscritos de Lacunza, hasta las primeras décadas del siglo XX. En este período, muchos sacerdotes, presbíteros y bachilleres se lanzan a apoyar o refutar el texto del jesuita, produciéndose una avalancha crítica, sobre todo enfocada sobre el acercamiento exegético de Lacunza. La interpretación del chileno se basa

${ }^{1}$ El presente trabajo forma parte de las actividades del Proyecto de Investigación DI 04/02-2, bajo la dirección del profesor Rolando Carrasco M. (Departamento de Literatura. Facultad de Filosofía y Humanidades, Universidad de Chile). 
en la literalidad como método para entender la Biblia, llegando así a su tesis acerca de dos juicios finales, y el interregno entre ambos, en la que Cristo, en persona, gobierna el mundo (reino milenario).

Textos representativos de esta etapa son los de Judas Tadeo Reyes (1820) y el de José Vidal (1834), en los que prima la discusión teológica. Estos debates teológicos-doctrinales (muchos de éstos llenos de acusaciones y descalificaciones) tienen como hito principal la inclusión de La Venida del Mesías en gloria y majestad en el Index romano en el año 1824.

La segunda etapa, que marca un fuerte debate acerca de la persona y obra del chileno, está situada en las primeras décadas del siglo XX. Autores como Emilio Vaisse (1917) y Miguel Rafael Urzúa (1917) discuten, no tanto sobre el apego de las ideas de Lacunza al catolicismo, sino que acerca de la originalidad del pensamiento del jesuita y sus postulados milenaristas. Toda esta querella gira en torno al rescate de la obra del jesuita en la época del centenario de Chile como república independiente (1910), momento histórico en el que existió una fuerte preocupación acerca del tema identitario.

A raíz de esto, se busca reafirmar la "chilenidad" con figurales culturales de categoría, como el caso de Manuel Lacunza.

La última etapa se inaugura con la publicación del estudio de Alfred-Félix Vaucher, Une célébrite oubliéé. Le P.M. Lacunza (1941). Este trabajo renueva la escena sobre los estudios acerca del jesuita chileno, ya que impulsa el interés de la crítica, que va recuperando el pensamiento de Manuel Lacunza, debido al tratamiento del jesuita sobre el tema apocalíptico (idea de sumo interés intelectual y colectivo a finales del siglo XX). Esta labor investigativa e interpretativa es continuada hasta hoy en día por varios estudiosos chilenos, por entre otros, Freddy Parra y Miguel Rojas Mix.

El presente estudio bibliográfico tiene como objetivo recopilar y comentar la mayor cantidad de trabajos disponibles que se han publicado sobre la figura y obra de Manuel Lacunza, para darlos a conocer a los estudiosos del período colonial para, de esta manera, facilitar y profundizar el conocimiento sobre este tema. La bibliografía recopilada ha sido dividida en dos partes: la primera incluye las referencias sobre las ediciones existentes de La Venida del Mesías en gloria y majestad y los estudios bio-bibliográficos que se han hecho sobre el jesuita. La segunda parte contiene las referencias sobre la bibliografía general en torno a la obra de Manuel Lacunza, divididas entre libros, artículos, por un lado; y reseñas, por el otro. En este trabajo también se presenta una bibliografía comentada, la cual corresponde a una selección de los estudios más relevantes sobre la obra del autor chileno. 


\section{BIBLIOGRAFIA GENERAL}

Fuentes Primarias

- $\quad$ Ediciones de La Venida del Mesías en gloria y majestad

Lacunza, Manuel. La Venida del Mesías en gloria y majestad. (4 Tomos). Londres: Ed. C. Wood, 1816.

Lacunza, Manuel. La Venida del Mesías en gloria y majestad. (Edición de P. de Chamrobert). París: Parmentier, 1825.

Lacunza, Manuel. La Venida del Mesías en gloria y majestad. (Selección de Emilio Vaisse). Santiago de Chile: Universitaria, 1915.

Lacunza, Manuel. La Venida del Mesías en gloria y majestad. (Edición antologada por Mario Góngora). Santiago de Chile: Universitaria, 1969.

Lacunza, Manuel. Tercera parte de La Venida del Mesías en gloria y majestad. (Edición preparada por Adolfo de Nortdenflycht). Madrid: Editora Nacional, 1978.

\section{Cartas de Manuel Lacunza}

Espejo, Juan Luis. "Cartas del padre Manuel Lacunza". Revista Chilena de Historia y Geografía. (Santiago de Chile). Tomo IX. N 13, (1914): 200219.

\section{Estudios Bio-BIBLIOGRÁFICOS}

Furlong, Guillermo. "Las ediciones castellanas del libro de Lacunza". Estudios (Buenos Aires). Tomo XXXIV (1928):144-150.

Schaible, Carl H. "Las primeras ediciones del padre Lacunza". Revista Chilena de Historia y Geografía (Santiago de Chile) no 111 (1948): 205-273.

Silva Castro, Raúl. "En torno a la bibliografía de Lacunza". Revista Chilena de Historia y Geografia (Santiago de Chile) nº 105 (1944): 167-182. 
Vaucher, Alfred. Une celebrité oubliée. Le P. Manuel Lacunza y Díaz. Collonges-sous-Salève: Fides, 1941 ( $1^{\mathrm{a}}$ ed.) y 1968 ( $2^{\mathrm{a}}$ ed.).

Zeballo, Ana de. "La Venida del Mesías de Manuel Lacunza. Primeras ediciones y críticas". Anuario de historia de la iglesia, ISSN 1133-0104, n 11, (2002): 115-128.

\section{ESTUDIOS SOBRE LACUNZA}

Agier P. J. Vues sur le second avenment de J.C. ou Analyse de l'ouvrage de M. Lacunza. París: Eberhart et Méquignon, 1818.

Anzoátegui, Víctor / Enrique Sanhueza Beltrán. Vulgarización de Lacunza y el Lacuncismo. Santiago: Universitaria, 1965.

Arteaga, J. “Temas apocalípticos y lacunzismo: 1880-1918”. Anales de la Facultad de Teología, PUCCh. (Santiago de Chile). Vol. XXXIX (1988): 209-224.

Bestard, Juan Buenaventura. Observaciones que Fr. Juan Buenaventura Bestard ... presenta al público, para precaverle de la seducción que pudiera ocasionarle la obra intitulada "La venida del Mesías en gloria y magestad" de Juan Josaphat Benezra. Madrid: Impr. de D. Fermin Villalpando, 18241825. (2 Tomos).

Bulnes Aldunate, Manuel. "Manuel Lacunza: contenidos teológicos y filosóficos de su interpretación profética". CEHILA (Costa Rica). (1985): 97-117.

Calderón de Puelles, Mariana. "Contado con los malvados: retórica y milenarismo en Manuel Lacunza". Anales de literatura chilena. Año 2, nº 2 (dic. 2001): 51-64.

Chaneton, Abel. En torno a un "papel anónimo» del siglo XVIII ciudad. Buenos Aires: J. Peuser, 1928.

Daneri, Juan José. "Los usos de la profecía: Escatología y política en $\mathrm{La}$ Venida del Mesías en gloria y majestad (1812) de Manuel Lacunza”. Silabario, $\mathrm{n}^{\mathrm{o}} 3$, junio, (2000): 91-100. 
Donoso, Ricardo. "La prohibición del libro del Padre Lacunza". Revista Chilena de Historia y Geografía (Santiago de Chile). N 135 (1967): 110-148.

Farías, Miguel Angel. "Lo americano en el texto La venida del mesías en gloria y majestad, de Manuel Lacunza". Logos n 1 (2ºmestre 1989): pp. 13-21.

Fernández, Maximino. "Algunos rasgos literarios en la obra de Manuel Lacunza”. Revista Universitaria (Santiago de Chile). N 51(1996): 45-48.

Furlong, Guillermo. "A propósito de Lacunza. Apostillas a un estudio del señor Abel Chaneton". Estudios (Buenos Aires). Tomo XXXVI, N 36 (1928): 3-15.

Giordano Jaime. Manuel Lacunza: un apocalipsis ilustrado. Actas Irvine (UCI), vol. 3 (1994): 263-270

Góngora, Mario. "La obra de Lacunza en la lucha contra el 'Espíritu del Siglo' en Europa 1770-1830”. Revista Historia nº 15 (1980); pp. 7-65.

"Un memorial de Lacunza". Revista Chilena de Historia y Geografía, (1955): 147-151.

González, Ruben. "Un ilustre editor de Manuel Lacunza: El general Manuel Belgrano". Boletín de la Academia Chilena de la Historia (Santiago de Chile), año 22, no 52 (1955): 149-158.

Guzmán, Alejandro. "La interpretación de Manuel Lacunza a la profecía de Daniel sobre los cinco reinos". Boletín de la Academia Chilena de la Historia no 100 (1989): 163 - 171.

Hanisch, Walter. "El Padre Manuel Lacunza (1731-1801), su hogar, su vida y la censura española". Revista Historia $\mathrm{n}^{\circ} 8$ (1969): 157-232.

"Lacunza o el temblor apocalíptico". Historia (Santiago de Chile), $\mathrm{N}^{\circ} 21$ (1986): 335-378.

Lazo, Olegario. "Lacunza y su Obra, por el Pbo. Miguel Rafael Urzúa". Revista Católica no18 (1914): 448-497; 569-578; 652-656; 725-728; y 19(1915): 50-54 
Lida, Raimundo / Emma Speratti. "Lacunza en México". Iberoamericana (Pittsburg) Vol. XVIV, N¹04-105 julio-diciembre (1978): 527-533.

Martínez, Frédéric. "Milenarismo y defensa de la fe en el Siglo de las Luces: la obra del jesuita chileno Miguel Lacunza". Revista Historia Crítica (Universidad de los Andes, Bogotá) no 3 enero-junio (1990): 41-62.

Mateos, Francisco. "El P. Manuel Lacunza y el Milenarismo". Revista Chilena de Historia y Geografia 115 (1950): 134-161.

"Milenarismo mitigado. Méritos y errores de un insigne jesuita chileno". Razón y Fe (Madrid) no 127 (1943).

Millar Carvacho, René: "Recepción de Lacunza en chile". $A H L G \mathrm{n}^{\circ} \mathrm{XI}$, (2002): 129-140.

Morales, Eddie: "Lacunza y el milenarismo: lectura de La Venida del Mesías en gloria y majestad". Ponencia X Congreso Internacional de Estudios Literarios (SOCHEL), Santiago, Universidad de Chile (1999).

"Lacunza y el discurso milenarista en la literatura colonial: La venida del Mesías en gloria y majestad". Nueva Revista del Pacifico, Facultad de Humanidades, Upla., $n^{\circ} 45,(2000)$.

Olivares, Sergio. “ Manuel Lacunza: la conexión adventista”. Diálogo Universitario (revista internacional de fe, pensamiento y acción). http://dialogue. adventist.org/articles/06_1_olivares_s.htm

Parra, Fredy. "El reino que ha de venir. Historia y esperanza en la obra de Manuel Lacunza". Anales de la Facultad de Teología. PUCCh (Santiago de Chile). XLIV (1993).

"El fin del mundo según Lacunza" En: Teología y Vida. Vol. XLI (2000); pp. 64-80.

"Historia y esperanza en la obra de Manuel Lacunza". Teología y Vida. Vol. XXXV (1994): 135-152.

"Manuel Lacunza: su obra y su visión de la historia". La Revista Católica $\mathrm{n}^{\circ} 1.133$ (2002): 29-38. 
"Historia y escatología en Manuel Lacunza: La temporalidad a través del milenarismo lacunziano". Teología y Vida. Vol. 44, N²-3 (2003): 163-187.

"La esperanza del reino en la obra de Manuel Lacunza". Tópico 90. no 4 (nov. 1992): 159-175

"El milenarismo y Manuel Lacunza". Revista Universitaria (Santiago de Chile) no 51, (1996) 39-44.

Priora, Juan Carlos. "Manuel Belgrano y Manuel Lacunza S. J.: Una conexión intelectual enigmática". Ponencia jornada de investigación 2002, Universidad Adventista del Plata (Argentina).

Ramos Mejía, Francisco (1819): "Notas a la obra de Manuel Lacunza”, en Clemente Ricci, "Francisco Ramos Mexía (Un heterodoxo argentino como hombre de genio y como precursor)". La Reforma, Revista Argentina de Religión, Historia, Ciencias Sociales y Revista de Revistas (Buenos Aires), 1923.

Reyes, Judas Tadeo de: Impugnación a la obra del Padre Manuel Lacunza sobre el reino milenario titulada La Venida del Mesías en gloria y majestad. 1820 (sin más datos).

Ricci, Clemente. Francisco Ramos Mexia y el Padre Lacunza. Buenos Aires: Impr. Kidd, 1929.

Rojas Mix, Miguel. El fin del milenio y el sentido de la historia. Manuel Lacunza, Juan Ignacio Molina. Santiago: LOM Ediciones, 2001.

Sasso, Marcello. "Góngora Mario. La obra de Lacunza en la lucha contra el 'Espíritu del siglo' en Europa”. Revista Chilena de Historia y Geografía (Santiago de Chile) no 149 (1981): 284-287.

Saranyana, Josep Ignasi. El milenarismo lacunciano y la teología de la liberación. $A H L G, \mathrm{n}^{\circ} \mathrm{XI},(2002): 141-149$

Seperiza Pasquali, Iván. "Lacunza el milenarista". Ciberscrito nº 65, Mundo Mejor: www.isp.co.cl/lacunza.htm 
Teólogo mexicano (anónimo). Disertaciones crítico-teológicas sobre las doctrinas de Juan Josafat Ben Ezra en su obra titulada La venida del Mesías en gloria y majestad. México: Imprenta de Luis Abediano y Valdés, 1848.

Unzueta Castillo, Luis. "Los temas en las cartas del padre Manuel Lacunza". En: www.uchile.cl/facultades/filosofía/cestculturales/lacunza/html

Urzúa, Miguel Rafael. Las doctrinas del padre Manuel Lacunza contenidas en su obra La venida del Mesías en gloria y majestad. Santiago: Universo, 1917.

"El Pbro. D. Emilio Vaisse y el lacunzismo". Revista Chilena de Historia y Geografia (Santiago de Chile). Tomo XI - XII (1917): 3-35.

Respuesta al Pbro. D. Olegario Laso por su artículo Lacunza y su obra. Santiago: Impr. San José, 1915. (Nota Separata de la Revista Católica $\mathrm{n}^{\mathrm{o}} 328$.)

“El R. P. Manuel Lacunza (1731-1801) y su obra 'La Venida del Mesías en gloria y majestad' (Londres, 1826), con motivo del primer aniversario de su muerte. Revista Chilena $\mathrm{n}^{\circ} 1$ y 2, (1901).

El padre Lacunza y su obra la venida del Mesías. Santiago: Imprenta Universitaria, 1914.

El R. P. Manuel Lacunza (1731-1801) y su obra "La Venida del Mesías en gloria y majestad" (Londres, 1826). Revista Chilena de Historia y Geografia (Santiago de Chile), v. 11-12, 1914.

Vaucher, Alfred-Félix. Lacunza, un heraldo de la Segunda Venida de Cristo. California-U.S.A: Interamericanas, 1970.

Vaisse, Emilio. "El P. Lacunza: Sus principales errores". Revista Chilena de Historia y Geografía (Santiago de Chile) nº 129 (1961): 14-22.

El lacunzismo. Santiago de Chile: Universitaria, 1917.

"Documentos inéditos. Cartas de Monseñor Muzi y de su secretario José Sallusti sobre la doctrina del padre Lacunza". Revista Chilena de Historia y Geografía (Santiago de Chile) no 27, 1917. 
Valdivieso, José. "Carta apologética en defensa de la obra de Juan Josafat Ben Ezra", incluido como Apéndice al tomo 3 de La venida del Mesías en gloria y majestad (London: Carlos Wood, s. f.), p. 332.

Vidal, José. Compendio de la impugnación de la obra titulada: Venida del Mesias en gloria y magestad. Dada a luz por el abate Lacunza. Lima: Imprenta.de José Maria Masias, 1838.

Vidal, Giuseppe. La venuta del Messia in gloria e maesta': osservazioni del sig. Ab. Lacunza ... / richiamate ad esame dal P. Giuseppe Vidal. Roma: Dalla Tipografia Salviucci, 1834.

Villegas, B. "El milenarismo y el Antiguo Testamento a través de Lacunza”. Dissertatio ad lauream in Facultates. Theologiae apud Pontificium Institutum "Angelicum” de urbe Valparaíso, 1951.

\section{RESEÑAS DESTACADAS EN DIARIOS Y REVISTAS}

Couyoumidjian, Ricardo. "Manuel Lacunza y Juan Ignacio Molina, dos perspectivas divergentes". Revista de libros. El Mercurio, 7-10-2001 (Reseña del libro El fin del milenio y el sentido de la historia de Manuel Rojas Mix).

Hanisch Walter. "El padre Manuel Lacunza”. El Mercurio (Santiago, Chile), mayo 17,1970 , p. 7.

Hanisch, Walter. "Manuel Lacunza y el milenarismo". El Mercurio (Santiago, Chile), mayo 14, 1972, p. 2.

Hernández, Roberto. "Molina y Lacunza [artículo]". El Mercurio (Santiago, Chile), agosto 14, 1980, p. 16 (suplemento).

Lefevre, Alfredo. "La venida de Manuel Lacunza [artículo]". Las Últimas Noticias (Santiago, Chile), abril 29, 1975, p. 2 (suplemento).

Lewin, Boleslao. "El Gral. Belgrano y el mesianismo del Padre Lacunza". La Prensa (Argentina), 14 de julio de 1870.

Parra, Fredy. "El regreso de Jesucristo. Crítica y actualidad de Lacunza". Artes y Letras, El Mercurio, 17 de junio de 2001, E-12 y E-13. 
Pico, José María. "Francisco Ramos Mexía y el Padre Lacunza". La Reforma (Argentina), mayo 1929.

Rolle, Claudio. "Vida de Manuel Lacunza: un jesuita utópico". El Mercurio 17-06-2001. 


\section{SELECCIÓN BIBLIOGRÁFICA COMENTADA}

Valdivieso, José. "Carta apologética en defensa de la obra de Juan Josafat Ben Ezra", incluido como Apéndice al tomo 3 de La venida del Mesías en gloria y majestad (London: Carlos Wood, s. f.), p. 332.

Breve carta que Valdivieso realiza para defender la obra de Lacunza de los ataques realizados por religiosos que consideran La Venida del Mesías en gloria y majestad como un texto lleno de infamias y de carácter heterodoxo.

Agier P. J. Vues sur le second avenment de J.C. ou Analyse de l'ouvrage de M. Lacunza. París: Eberhart et Méquignon, 1818.

Este escrito de Agier, en francés, realiza la labor de recapitulación de los contenidos de la obra del chileno Manuel Lacunza, La Venida del Mesías en gloria y majestad. El autor, una autoridad jansenista, busca informar y difundir las teorías del jesuita, para que fueran conocidas y estudiadas.

Reyes, Judas Tadeo de. Impugnación a la obra del Padre Manuel Lacunza sobre el reino milenario titulada La Venida del Mesías en gloria y majestad. 1820 .

Esta es la primera obra crítica que aparece después de la publicación formal de La Venida de el Mesías en gloria y majestad.

En este trabajo, el autor busca hacer una impugnación a algunos aspectos de la obra de Lacunza, desde una perspectiva absolutamente católica ortodoxa.

La principal crítica de este escrito es al método exegético que utiliza el jesuita, privilegiando el sentido literal, alejándose, según él, demasiado de las doctrinas del catolicismo.

Bestard, Juan Buenaventura. Observaciones que Fr. Juan Buenaventura ... presenta al público, para precaverle de la seducción que pudiera ocasionarle la obra intitulada La venida de Mesias en gloria y magestad de Juan Josaphat Benezra. Madrid: Impr. de D. Fermin Villalpando, 1824-1825. (2 Tomos).

Esta extensa obra de Bestard tiene la intención explícita de refutar la obra de Manuel Lacunza. A partir de largas citas bíblicas, rechaza el método exegético del jesuita, y critica su desprecio a las opiniones de los doctores de la iglesia. El análisis de la obra La Venida del Mesías en Gloria Majestad por parte de 
Bestard incluye fuertes calificativos, como "veneno mortífero" (refiriéndose al contenido del texto), o al mismo Lacunza como "sofista".

Vidal, José. Compendio de la impugnación de la obra titulada: Venida del Mesias en gloria y magestad. Dada a luz por el abate Lacunza ... / llamada a examen por el R.P.F. José Vidal, impresa en Roma en lengua italiana, el año de 1834 ; traducida al castellano por el R.P. Fr. Andrés Herrero y compendiada por el R.P. F. Angel Vicente de Zea. Area de Publicación Lima : Imprenta de José María Masias, 1838. Descripción vi, [6], 272 p. (Biblioteca Nacional)

La obra de José Vidal está enfocada en el análisis de la obra La Venida de el Mesías en gloria y majestad en forma detallada, buscando hacer alguna impugnación al texto. La conclusión a la que llega Vidal sobre el libro del jesuita es que es un texto que contiene ideas milenaristas, por lo tanto, el chileno sería un heterodoxo.

Teólogo mexicano (anónimo). Disertaciones crítico-teológicas sobre las doctrinas de Juan Josafat Ben Ezra en su obra titulada La venida del Mesías en gloria y majestad. México, Imprenta de Luis Abediano y Valdés, 1848.

Este anónimo mexicano busca reivindicar la obra La Venida del Mesías en gloria y majestad, fuertemente atacada por el ambiente teológico católico de las primeras décadas del siglo XIX.

El teólogo anónimo rechaza las opiniones de los religiosos que sin mayores argumentos denostan la obra de Lacunza, obra que según el mexicano es impecable. En la primera parte del texto defiende el método exegético del chileno, y en la segunda responde a las objeciones hechas por Bestard y el doctor Alfaro.

Urzúa, Miguel Rafael. El R. P. Manuel Lacunza (1731-1801) y su obra La Venida del Mesías en gloria y majestad (Londres, 1826), con motivo del primer aniversario de su muerte. Revista Chilena $\mathrm{n}^{\circ} 1$ y 2, 1901.

Este texto inaugura una nueva era en la discusión sobre la obra de Manuel Lacunza. Urzúa hace aquí una recuperación y una apología sobre la olvidada vida y obra del jesuita chileno. Defiende con ahínco los ataques que le hacen los teólogos católicos, y afirma que nadie ha probado falsedad o errores en La Venida del Mesías en gloria y majestad. Y termina con la idea 
de que esta obra solo fue prohibida por simple cautela, por el temor a malas interpretaciones, y no porque la obra estuviera mal en sí.

Urzúa, Miguel Rafael. El R. P. Manuel Lacunza (1731-1801) y su obra $L a$ Venida del Mesías en gloria y majestad (Londres, 1826), Revista Chilena de Historia y Geografia (Santiago de Chile), v. 11-12, 1914

Urzúa, Miguel Rafael. El padre Lacunza y su obra la venida del Mesías. Santiago: Imprenta Universitaria, 1914.

Estas dos publicaciones están basadas en el texto de 1901, continuando con el rescate de la figura y obra de Lacunza. Se hace una reseña biográfica, se resume y se analizan las ideas centrales del texto del jesuita y su legado.

Juan Luis Espejo. "Cartas del padre Manuel Lacunza". Revista Chilena de Historia y Geografia (Santiago de Chile). Tomo IX, n 13 (1914); pp. 200219.

Espejo nos entrega un estudio biográfico sobre Lacunza en donde aparecen cuatro cartas inéditas escritas por el autor de La Venida del Mesías en gloria y majestad, desde su exilio en Imola, Italia. El autor esboza una biografía del jesuita chilena y busca llenar los vacíos acerca de su vida mediante la presentación de estas cuatro cartas, las que vienen reproducidas en su totalidad.

Lazo, Olegario. "Lacunza y su Obra, por el Pbo. Miguel Rafael Urzúa". Revista Católica 18 (1914), 448-497; 569-578; 652-656; 725-728; y 19, 50-54 (1915).

Este trabajo surge como respuesta a la defensa de Urzúa de los postulados de Lacunza. Lazo realiza un fuerte ataque a los contenidos de La Venida del Mesías en gloria y majestad, considerándolos como erróneos y peligrosos. Además, para el autor, la interpretación de la Biblia por el jesuita carece del suficiente conocimiento lingüístico para analizar ciertas palabras clave para llegar al sentido correcto.

Urzúa, Miguel Rafael. Respuesta al Pbro. D. Olegario Laso por su artículo Lacunza y su obra. Santiago: Impr. San José, 1915 (Nota Separata de la Revista Católica $\mathrm{n}^{\circ} 328$ ). 
En la respuesta a los ataques de Olegario Lazo a La Venida de el Mesías en gloria y majestad, Urzúa replica con los mismos argumentos esgrimidos con antelación, insistiendo en la validez de las teorías de Lacunza, su apego a las Escrituras, su erudición privilegiada y su originalidad.

Miguel Rafael Urzúa. "El presbítero don Emilio Vaisse y el lacunzismo. Revista Chilena de Historia y Geografía (Santiago de Chile). Tomo XI-XII (1917), pp. 3-35.

En este trabajo, Urzúa realiza una fuerte crítica a Emilio Vaisse sobre su análisis de la persona de Manuel Lacunza y su obra La Venida del Mesías en gloria y majestad. El autor acomete descalificatoriamente la visión negativa de Vaisse con respecto al jesuita chileno, ya que la encuentra injusta y antojadiza. Urzúa realiza un defensa de Lacunza, refutando las acusaciones tanto de Vaisse como de Menéndez Pelayo, poniendo énfasis en el reconocimiento mundial que tiene el jesuita, la originalidad y su apego a las Escrituras. Urzúa recalca la misión de rescatar la figura y obra de Manuel Lacunza, ya que, según él, es uno de las nombres del pensamiento nacional que mayor trascendencia ha tenido fuera de nuestras fronteras

Urzúa, Miguel Rafael. Las doctrinas del padre Manuel Lacunza contenidas en su obra La venida del Mesías en gloria y majestad. Santiago: Universo, 1917.

Este texto de Miguel Rafael Urzúa busca hacer una recuperación y una defensa de la obra La Venida del Mesías en Gloria Majestad, de Manuel Lacunza, desde una perspectiva católica y militante. El autor hace un minucioso estudio, a partir de extractos escogidos de la obra, de los postulados del jesuita, con la intención de defenderlos contra los ataques que eran realizados por autores como, por ejemplo, Emilio Vaisse.

Vaisse, Emilio. El lacunzismo. Santiago de Chile: Universitaria, 1917.

En este trabajo, Vaisse busca probar la poca originalidad de la doctrina de Manuel Lacunza, rebatiendo a los que consideran la obra del chileno como una obra maestra y de un legado enorme.

Para probar su punto, el autor realiza un estudio de la corriente milenarista, y rastrea cómo influye en la doctrina del jesuita. También busca demostrar 
la escasa influencia de la obra de Lacunza en los movimientos religiosos posteriores, localizando esta influencia en pequeñas iglesias protestantes. El libro incluye además una lista de apuntes bibliográficos sobre el milenarismo moderno.

Vaisse, Emilio. Documentos inéditos. Cartas de Monseñor Muzi y de su secretario José Sallusti sobre la doctrina del padre Lacunza. Revista Chilena de Historia y Geografía (Santiago de Chile) n² 27, 1917.

Estas cartas sobre Lacunza aparecen también en el libro de Vaisse El lacunzismo, donde se incluyen como documentos inéditos. Estas cartas están dirigidas a Judas Tadeo de Reyes, quien realizó una impugnación a la obra La Venida. Las cartas de Muzi y Sallusti son básicamente para felicitar a Reyes por su impugnación del texto del chileno.

Ramos Mejía, Francisco (1819). "Notas a la obra de Manuel Lacunza”, en Clemente Ricci, "Francisco Ramos Mexía (Un heterodoxo argentino como hombre de genio y como precursor)", La Reforma, Revista Argentina de Religión, Historia, Ciencias Sociales y Revista de Revistas (Buenos Aires), 1923.

El argentino Ramos Mejía realiza un resumen, y un análisis de carácter positivo sobre la figura y la obra de Manuel Lacunza, en el cual se inspirará para producir sus propias doctrinas, de carácter adventista.

Abel Chanetón. En torno a un papel anónimo del siglo XVIII. Buenos Aires: Talleres Casa Jacobo Peuser, 1928.

Este estudio trata acerca de un papel anónimo llamado "Papel anónimo sobre la segunda venida de Christo”, el que circuló a finales del siglo XVIII, atribuido al jesuita chileno Manuel Lacunza. Chanetón sostiene que este documento era un resumen del libro La Venida del Mesías en gloria y majestad, el cual, a pesar de no haber sido publicado, ya contaba con muchas copias manuscritas, tales como el "Papel anónimo". El autor se centra en la impugnación (según Furlong la primera de que se tenga noticia) que hace en 1787 el argentino Dalmacio Vélez a este papel, sus motivaciones económicas para esta empresa y su nula recepción en la región del Río de la Plata, en la que el pensamiento de Lacunza había tenido una favorable acogida. Además, el autor plantea que los manuscritos circulantes de Lacunza responden 
a un interés político de la Compañía de Jesús, recientemente expulsada de los dominios americanos. Se incluye también en este trabajo el documento llamado "Papel anónimo sobre la segunda venida de Christo"(que el autor atribuye totalmente a Lacunza), y el documento "Impugnación al Papel anónimo sobre la segunda venida de Christo” de Dalmacio Vélez.

Guillermo Furlong. "A propósito de Lacunza. Apostillas a un estudio del señor Abel Chanetón". Estudios (Buenos Aires). Tomo XXXVI, n 36 (1928); pp. 3-15.

Este artículo intenta refutar el trabajo de Abel Chanetón, llamado En torno a un papel anónimo del siglo XVIII. Furlong critica el hecho que Chanetón afirme que ese "papel anónimo" sea verdaderamente de Lacunza, puesto que es una copia pésima y desfigurada de la gran y extensa obra de Lacunza, según el autor. También se opone a la idea de Chanetón, la cual esgrime que la Compañía de Jesús habría hecho circular copias manuscritas de la obra del chileno con fines políticos. El autor recuerda que la misma orden jesuita fue la que más condenó la obra La Venida del Mesías en gloria y majestad (además Furlong sostiene que los manuscritos circulantes eran muy pocos). El autor acusa a Chanetón de calumniar a la Compañía de Jesús, realizando generalizaciones absurdas y de mala intención.

Guillermo Furlong. "Las ediciones castellanas del libro de Lacunza". Estudios (Buenos Aires). Tomo XXXVI, septiembre-diciembre, 1928, pp.144-150.

En este artículo se hace una descripción de todas las ediciones existentes desde 1818 hasta 1928 de La Venida del Mesías en gloria y majestad, con sus respectivos datos técnicos, editoriales, además de algunas noticias importantes acerca de cada una de las ediciones.

Clemente Ricci. Francisco Ramos Mexia y el padre Lacunza. Buenos Aires, Impr. Kidd, 1929.

Este autor discute acerca de la real influencia que ejerció la obra de Manuel Lacunza, La Venida del Mesías en gloria y majestad, en el argentino Francisco Ramos Mexia.

Ricci se opone a la afirmación de Abel Chanetón, el cual postula que Lacunza es el gran guía de Ramos Mexía. El crítico afirma que el argentino se relaciona con la obra del jesuita chileno, porque mucho de sus propios 
postulados concuerdan con del autor de la Venida. Eso sí, afirma el crítico, que el propio Ramos tenía el deseo de diferenciarse de Lacunza, enfatizando sus discrepancias. En este sentido, recalca Ricci, la visión adventista de Ramos es anterior a Lacunza, pero no puede explicar el origen de este adventismo más que en una "intuición genial" por parte del argentino.

Mateos, Francisco. "Milenarismo mitigado. Méritos y errores de un insigne jesuita chileno". Razón y Fe no 127 (1943).

Es este estudio, Francisco Mateos plantea que el jesuita chileno Manuel Lacunza tiene muchos errores de interpretación sobre las escrituras en su obra La Venida del Mesías en gloria y majestad, pero no duda de su calidad de buen católico. Es más, considera que los desaciertos de Lacunza son a causa de los problemas psicológicos que le provocó el destierro.

Raúl Silva Castro. "En torno a la bibliografía de Lacunza". Revista Chilena de Historia y Geografia (Santiago de Chile) nº 105, 1944; pp.167-182.

En este trabajo Silva Castro intenta clarificar el orden de las tres primeras ediciones de La Venida del Mesías en gloria y majestad, que se publican en España bajo el nombre del editor Felipe Tolosa. Primero el autor se opone a la afirmación de José Toribio Medina, el que postula que la edición con la advertencia de Tournachon-Molin es la primera publicada.

Silva Castro relega a esta edición como la tercera, basándose en la cita de ella que hace el francés fray Buenaventura Bestard, quien afirma que no es la primera edición. Concluye el estudioso en que la primera edición de $L a$ Venida del Mesías en gloria y majestad fue efectivamente en España, y que la edición considerada la primera por Medina y Vaisse, es en realidad, la tercera.

Schaible, Carl H. "Las primeras ediciones del padre Lacunza". Revista Chilena de Historia y Geografía (Santiago de Chile) no 111 (1948); pp. 205-273.

Este trabajo realiza una exhaustiva investigación sobre las fechas y lugares de publicación de las primeras ediciones de la obra La Venida del Mesías en gloria y majestad. Schaible se dedica a presentar y a discutir las diferentes hipótesis acerca de la datación de las primeras ediciones del texto del jesuita. A partir también de su propia investigación, el autor propone fechas y lugares 
sobre las cuatro primeras publicaciones, como por ejemplo, situar la primera edición en el año 1811, en la isla de León, en España.

Mateos, Francisco. "El P. Manuel Lacunza y el Milenarismo”. Revista Chilena de Historia y Geografía, 115 (1950); pp. 134-161 (Biblioteca Nacional).

En este estudio, el autor analiza las doctrinas que aparecen en la obra de Manuel Lacunza, La Venida del Mesías en gloria y majestad, para conectarlas con las ideas de la corriente de pensamiento llamada Milenarismo, encontrando que el jesuita chileno se ve influido por la tendencia milenaria para realizar su lectura sobre el reino terrenal de Cristo sobre la tierra.

Villegas, B. El milenarismo y el Antiguo Testamento a través de Lacunza: dissertatio ad lauream in Facultates. Theologiae apud Pontificium Institutum "Angelicum" de urbe Valparaíso, 1951.

En este trabajo, el autor se dedica a investigar, a partir de los postulados de Manuel Lacunza, el tema del milenarismo.

El análisis que hace Villegas sobre el Antiguo Testamento, (ampliamente utilizado por Lacunza) lo lleva a afirmar que no hay ningún elemento textual que le dé validez a la interpretación milenarista, siendo el reino mesiánico intermedio una entidad que no aparece en las Escrituras.

Rubén González. "Un ilustre editor de Lacunza: el general Manuel Belgrano". Boletín de la Academia Chilena de la Historia (Santiago de Chile). Año 22, 1955, n 52; pp. 149-158.

Este artículo se refiere a la participación de Manuel Belgrano en la publicación de 1816 del libro La Venida del Mesías en gloria y majestad, de Manuel Lacunza. La admiración hacia Lacunza en la región rioplatense lleva al general Manuel Belgrano a publicar durante su estada en Londres la obra del chileno, en la imprenta de Carlos Wood.

Este autor sostiene que fue Belgrano la persona impulsora de la edición londinense y no otros personajes que los estudiosos han propuesto, como el caso del argentino Manuel Moreno o el español José Joaquín de Mora. La postura de González se apoya en la carta de fray Cayetano Rodríguez al presbítero José Agustín Molina, y por sobre todo en el testimonio del canónigo Juan Ignacio Gorriti, quien afirma que el general argentino es el verdadero financista de la publicación de Lacunza. 
Emilio Vaisse. "El padre Lacunza: sus principales errores". Revista Chilena de Historia y Geografía (Santiago de Chile) no 129 (1961), pp. 14-22.

En este artículo, Emilio Vaisse mantiene su antigua tesis (1917) de que la obra de Lacunza no es tan original como se la cree en el ambiente intelectual.

También se extiende, con una marcada ironía, acerca del influjo del jesuita chileno en la llamada Iglesia Católica Apostólica, fundada por W. Irving, quien la funda a partir de las teorías de Lacunza. (Iglesia con pocos seguidores y con muchas profecías no cumplidas, según Vaisse). Después pasa a atacar directamente al autor de La Venida del Mesías en gloria y majes$\mathrm{tad}$, refiriéndose a las autoridades que lo han criticado o impugnado, tales como Judas Tadeo Reyes, monseñor Muzzo, Francisco Heinrich, el padre Muzannelli, el padre Zea, y el Presbítero Sallusti. Para acentuar los errores de Lacunza, Vaisse, concluye que las teorías del jesuita han tenido mayor recepción en círculos protestantes y en sectas heréticas, lo que confirmaría sus desaciertos teológicos.

Anzoátegui, Víctor / Enrique Sanhueza Beltrán. Vulgarización de Lacunza y el Lacuncismo. Santiago: Universitaria, 1965.

En este estudio, los autores realizan un estudio sobre la recepción, las críticas, y el influjo posterior de la obra de Manuel Lacunza, La Venida del Mesías en gloria y majestad. Se hace hincapié en los ataques injustificados sobre la obra del jesuita, además de sus malas interpretaciones a lo largo del tiempo.

Ricardo Donoso. "La prohibición del libro del padre Lacunza". Revista Chilena de Historia y Geografia (Santiago de Chile) n 135 (1967), pp.110-148.

Este artículo hace una historia acerca de la circulación y posterior publicación de la obra La Venida del Mesías en gloria y majestad, de Manuel Lacunza. Se contextualiza la producción del texto en la época del reinado de Carlos III, y su favorable recepción en Buenos Aires y en México. Su publicación en España con la llegada de Fernando VII se mira con sospecha, hasta que se prohíbe el texto de Lacunza por parte del Vaticano en 1824.

Alfred-Félix Vaucher. Une célébrite oubliéé. le P. Manuel de Lacunza y Diaz (1731-1801). Collonges-sous-Saleve: Imprimerie FIDES, 1968.

Este estudio de Vaucher es considerado por la crítica como el más importante acerca de la vida y obra del jesuita chileno Manuel Lacunza. Publicado en 
francés, este trabajo se dedica de manera exhaustiva a investigar los aspectos fundamentales de la vida y obra del chileno: una biografía de Lacunza y diversos aspectos de su obra: ediciones en español, en latín, y las traducciones en inglés y en italiano. También el adventista analiza el contenido de la obra (el título, el método exegético, el sistema teológico, entre otros puntos). Aparece estudiada la originalidad del pensamiento de Lacunza y sus posibles fuentes (del judaísmo, el joaquinismo, el protestantismo y el jansenismo). Otros aspectos analizados en este trabajo son las polémicas que suscitó la obra de Lacunza en todo el mundo. También tiene un capítulo dedicado a La Venida del Mesías en gloria y majestad y su juicio por la Inquisición, y por último, el autor analiza la influencia de Lacunza sobre los círculos católicos, judíos, y protestantes. Además contiene una abundante bibliografía sobre el jesuita chileno, y textos relacionados con la materia (milenarismo).

Hanisch, Walter. "El Padre Manuel Lacunza (1731-1801), su hogar, su vida y la censura española”. Revista Historia 8 (1969), pp. 157-232.

En este trabajo, Hanisch realiza una minuciosa investigación sobre la vida de Manuel Lacunza, describiendo y analizando sus orígenes, su formación, su vida religiosa, su destierro, su producción escritural, los efectos que provocaron sus ideas, y la posterior censura de la Inquisición sobre La Venida del Mesías en gloria y majestad.

Vaucher, Alfred-Félix. Lacunza, un heraldo de la Segunda Venida de Cristo. California-U.S.A: Interamericanas, 1970.

Este libro nace como una recopilación de artículos publicados por Vaucher en la reviste El centinela de 1967 a 1969. Básicamente contiene los capítulos de su famoso Une célébrite oubliéé.

Raimundo Lida / Emma Speratti. "Lacunza en México". Revista Iberoamericana (Pittsburg). Vol. XLIV, ${ }^{\circ}$ 104-105, julio-diciembre, 1978, pp. 527-533.

Este artículo se centra específicamente en los comentarios que recibió un documento llamado Discurso en que se manifiestan las falsedades y errores de Juan Josafat Ben Ezra acerca de la Iglesia Cristiana, escrito por el capellán Pedro Narciso Blanco, en las primeras décadas del siglo XIX. Los críticos nos indican que este documento jamás se publicó, ya que recibió severas 
críticas de sus evaluadores para su publicación, como las del bachiller José María Sartorio, y las del mercedario Manuel Mercadelli, ambas en 1824. Lida y Speratti nos exponen las objeciones de estos evaluadores para con el Discurso. El bachiller Sartorio dice que no le gustaría criticar una obra que la mayoría de los teólogos encontró excelente, además de que pone en duda la capacidad teológica del capellán Blanco. El mercedario Mercadelli afirma que Blanco solo buscó errores y falsedades en la obra, con razones e impugnación débiles. Debido a esto, Mercadelli impuso tantas correcciones al Discurso para su publicación que el escrito del capellán jamás llegó a la imprenta. Esto demostraría, en opinión de Lida y Speratti, la buena recepción que tuvo la obra de Manuel Lacunza, La Venida del Mesías en gloria y majestad en México.

Adolfo de Nortdenflycht. Introducción. Tercera parte de La Venida del Mesías en gloria y majestad de Manuel Lacunza (1978). Madrid: Editora Nacional, 1978.

Este trabajo es una introducción que busca realizar una panorámica de la vida del jesuita chileno en forma literaria. También hace un resumen de las doctrinas de Manuel Lacunza, mostrando la influencia que tiene la obra del jesuita chileno, entre otros puntos. Se incluyen, además, las Cartas del religioso.

Góngora, Mario. "La obra de Lacunza en la lucha contra el "Espíritu del Siglo" en Europa 1770-1830”. Revista Historia 15 (1980); pp. 7-65.

En este trabajo del historiador chileno Mario Góngora, la atención está puesta en relacionar el texto de Lacunza con algunas de las obras producidas en el siglo XVIII, obras que compartirían el rechazo a la ideas de la Ilustración, oponiéndoles, además, un sentido escatológico de la historia.

Autores como los españoles Hervás y Bruel, los jansenistas en Francia, Lewis Way en Inglaterra, Edward Irving en Escocia, Lavater y Jung-Stilling en Alemania, entre otros, desfilan como ejemplos de la lucha en contra del "Espíritu del siglo".

Góngora concluye que existió una atmósfera apocalíptica en aquella época que contribuyó a la creación de La Venida de el Mesías en gloria y majestad, y que esto colaboró con su posterior influjo. 
Sasso F., Marcello. "Góngora Mario. La obra de Lacunza en la lucha contra el "Espíritu del siglo" en Europa". Revista Chilena de Historia y Geografia (Santiago, Chile) no 149 (1981); pp. 284-287.

Este artículo de Sasso es sobre el estudio de Mario Góngora "La obra de Lacunza en la lucha contra el "Espiritu del Siglo". Primeramente, el autor hace un resumen de los planteamientos que expone Góngora sobre la obra de Lacunza y su relación con otras obras de la época con las que compartiría sentimientos apocalípticos anti-Ilustración. Para el autor, pese a encontrar impecable la obra de Góngora, éste exageraría la atmósfera apocalíptica que influiría en la escritura del jesuita chileno.

Bulnes Aldunate, Manuel. "Manuel Lacunza: contenidos teológicos y filosóficos de su interpretación profética". CEHILA (Costa Rica). 1985, pp. 97-117.

En este estudio, Bulnes, desde una óptica cercana a la teología de la liberación, recupera la visión crítica de Lacunza sobre el accionar de la Iglesia Católica, que se hallaba en momentos de degradación para el jesuita chileno. También el autor habla sobre los debates internos del muerto en Imola, escondidos bajo conceptos como profecía, reino terrenal, etc.

Hanisch, Walter. "Lacunza o el temblor apocalíptico". Historia (Santiago de Chile) $n^{\circ} 21$ (1986); pp. 335-378.

Walter Hanisch en este trabajo, analiza las 3 partes que componen la obra La Venida del Mesías en gloria y majestad, con el propósito de hacer un resumen de las doctrinas apocalípticas. La primera llamada "Tiempo intermedio", donde busca probar la existencia del tiempo intermedio, lugar que existe entre el juicio de los vivos y el juicio de los muertos. La segunda es referida a la interpretación de las figuras simbólicas (Daniel, Anticristo, la misión del pueblo judío), en los fenómenos y los anti-fenómenos. La tercera parte corresponde al análisis del llamado reino milenario, viendo aspectos como: el juicio de Cristo, la Nueva Jerusalén, entre otros.

Arteaga, J. "Temas apocalípticos y lacunzismo: 1880-1918”. Anales de la Facultad de Teología, PUCCh. (Santiago de Chile). Vol. XXXIX (1988), pp. 209-224. 
En este artículo, Arteaga se dedica a rastrear la presencia y la influencia del pensamiento de Lacunza en Chile entre el siglo XIX y las primeras décadas del XX.

En su análisis, revisa las referencias sobre el jesuita, sobre todo en el ámbito religioso e histórico. El autor se concentra en la evolución del pensamiento apocaliptico-milenarista para encontrar si Lacunza ha influenciado explícita o implícitamente a los pensadores chilenos de la religión.

Farías Farías, Miguel Ángel. "Lo americano en el texto La venida del mesías en gloria y majestad, de Manuel Lacunza". Logos $\mathrm{n}^{\circ} 1$ ( $2^{\circ}$ semestre 1989); pp. 13-21.

En este trabajo, Farías busca rastrear los componentes específicamente americanos de la obra La Venida del Mesías en gloria y majestad, utilizando, metodológicamente, la categoría llamada shifters, propuesta por Roland Barthes. A partir de esta noción, el autor reconoce como típicamente americano en el discurso de Lacunza, elementos como la censura, el exilio, y la idea de utopía.

Martínez, Frédéric. "Milenarismo y defensa de la fe en el Siglo de las Luces: la obra del jesuita chileno Miguel Lacunza". Revista Historia Crítica (Universidad de los Andes, Bogotá) no 3 enero-junio 1990, pp. 41-62.

El estudio de Martínez parte vinculando a Lacunza con el milenarismo, para después analizar su método exégetico y su visión teológica de la historia. Todo esto con el objetivo de demostrar que la obra de Lacunza (una doctrina teocéntrica escatológica, según el autor) es un defensa del cristianismo frente a la filosofía iluminista. Es más, para el autor, Lacunza trata de salvar la fe, viendo que la Iglesia estaba cediendo ante estos ideales ilustrados.

Parra, Fredy. "La esperanza del reino en la obra de Manuel Lacunza”. Tópico 90 n 4 (nov. 1992); pp. 159-175.

Parra, Fredy. "Historia y esperanza en la obra de Manuel Lacunza". Teología y Vida. Vol. XXXV (1994); pp. 135-152.

En estos artículos, Parra examina una problemática recurrente de la iglesia católica, la que tiene ver con el tema de la escatología, sobre todo en la conexión entre esperanza e historia. Para esto, el autor analiza la obra de Lacunza, en su calidad de pensador milenarista, revisando los siguientes 
puntos de su obra: el tema de la esperanza en Lacunza; la concepción de la historia; las características del reino de Cristo; la articulación entre lo humano y la esperanza, y por último, algunas consideraciones críticas.

Parra, Fredy. "El reino que ha de venir. Historia y esperanza en la obra de Manuel Lacunza". Anales de la Facultad de Teología. PUCCh. (Santiago de Chile). XLIV (1993).

Este trabajo de Parra está dominado por una perspectiva liberacionista, ya que se enfoca en la visión teológica de la historia que realiza Manuel Lacunza en su obra La Venida del Mesías en gloria y majestad. Para el autor, Lacunza es un ejemplo a seguir por su actitud justiciera y esperanzadora, pero por sobre todo, por su postura en contra del poder total y corruptor de la Iglesia, lo que se refleja en su escritura sobre el mundo milenario que vendrá.

Giordano Jaime. Manuel Lacunza: un apocalipsis ilustrado. UCI, Actas Irvine, vol 3, 1994, pp. 263-270.

En este trabajo, Giordano realiza un análisis discursivo de la ideas de Lacunza, tocando puntos como su lugar de enunciación, el destinatario del texto, el quiebre con los postulados tradicionales católicos. También se examina la función de la ironía, la utopía y la distopía en el discurso del jesuita chileno. Todo esto, para el autor, sería manifestación de rechazo a las nuevas ideas de la era ilustrada.

Priora, Juan Carlos. "Manuel Belgrano y Manuel Lacunza S.J.: Una conexión intelectual enigmática". Ponencia jornada de investigación 2002. Universidad Adventista del Plata (Argentina).

Esta investigación busca develar el interés del prócer argentino Manuel Belgrano en la obra de Manuel Lacunza. Para el autor, resulta extraño que ningún biógrafo de Belgrano se haya preocupado por este interés en publicar La Venida del Mesías en gloria y majestad. Priora propone cuatro hipótesis, no excluyentes, para explicar el afán del argentino en dar a conocer el texto del jesuita chileno.

Maximino Fernández. "Algunos rasgos literarios en la obra de Manuel Lacunza”. Revista Universitaria (Santiago de Chile) no 51 (1996); pp. 45-48. 
En este breve artículo, Fernández destaca algunas características literarias de las obras de Manuel Lacunza. El autor se aproxima desde una perspectiva estética-formal, haciendo hincapié en el estilo escritural del jesuita, el que se caracteriza por su sencillez, belleza, armoniosidad, y profundidad, las que se encuentran tanto en La Venida del Mesías en gloria y majestad, como en sus Cartas.

Freddy Parra. "El milenarismo y Manuel Lacunza". Revista Universitaria (Santiago de Chile) no 51 (1996); pp. 39-44.

Parra, en este artículo, parte haciendo una breve reseña del milenarismo, para luego ver los aspectos medulares de la doctrina lacunziana. El autor explica que Lacunza propone la teoría del tiempo intermedio, es decir, que Cristo reinará sobre la tierra mil años, desde el juicio a los vivos, hasta el juicio final de los muertos. También se expone el pensamiento del jesuita con respecto a la corrupción de la Iglesia, y la naturaleza moral del Anticristo. Parra se refiere además al pensamiento utópico social de Lacunza, ya que éste propone una transformación cósmica que traerá justicia, creando un mundo idílico

Morales, Eddie. "Lacunza y el milenarismo: lectura de La Venida del Mesías en gloria y majestad" (ponencia X Congreso Internacional de Estudios Literarios (SOCHEL). Santiago, Universidad de Chile, 1999 (Universidad de Chile).

Morales, Eddie. "Lacunza y el discurso milenarista en la literatura colonial: La venida del Mesías en gloria y majestad". Nueva Revista del Pacífico, Facultad de Humanidades, Upla., nº 45, 2000. (Biblioteca Nacional).

Estos trabajos de Morales se dedican a realizar un resumen de la obra de Manuel Lacunza, La Venida del Mesías en gloria y majestad. Posteriormente, asocian los postulados que aparecen en el texto con las ideas de la corriente religiosa milenarista, esto debido a que en la época colonial el pensamiento milenarista tuvo una gran influencia, sobre todo, según el autor, en Lacunza.

Parra, Fredy. "El fin del mundo según Lacunza”. Teología y Vida. Vol. XLI (2000); pp. 64-80.

Este artículo tiene como fin analizar el tema del fin del mundo en la obra $L a$ Venida del Mesías en gloria y majestad, concentrándose en la relación fin de mundo -mundo mejor que se expresa en el texto. Para el autor, Lacunza 
propone que el reino de Cristo en la tierra modificará todo para mejor, tanto a nivel físico, como a nivel espiritual.

Daneri, Juan José. "Los usos de la profecía: Escatología y política en La Venida del Mesías en gloria y majestad (1812) de Manuel Lacunza". Silabario $\mathrm{n}^{\mathrm{o}}$ 3, junio, 2000, pp. 91-100.

Daneri, Juan José. "Escatología y política jesuitas. La profecía del fin de los tiempos según Manuel Lacunza”. Mapocho 58 (2005): 181-201.

Estos trabajos de Daneri estudian la relación entre escatología y política en la obra La Venida del Mesías en gloria y majestad. Para el autor, existiría una relación muy estrecha entre el reino mesiánico milenario que propone Lacunza, y la política contingente de aquella época, es decir, el destierro de los jesuitas de los dominios americanos por parte de la monarquía española, con la anuencia de la Iglesia Católica.

Calderón de Puelles, Mariana. Contado con los malvados: retórica y milenarismo en Manuel Lacunza. Anales de Literatura Chilena. Año 2, $\mathrm{n}^{\mathrm{o}} 2$ (dic. 2001).

A partir de un enfoque retórico, la autora estudia la obra de Lacunza, $L a$ Venida del Mesías en gloria y majestad. Todo esto para proponer que en el texto del jesuita chileno, más que persuadir, busca atacar a sus enemigos (Iglesia, monarquía), funcionando esta obra como respuesta por su situación desmedrada. La estudiosa, para este trabajo, analiza la dedicatoria, el prólogo, el discurso preliminar y fragmentos de la obra,

Miguel Rojas Mix. El fin del milenio y el sentido de la historia. Manuel Lacunza y Juan Ignacio Molina. Santiago de Chile: LOM, 2001.

En este trabajo, Rojas Mix analiza la obra de Manuel Lacunza, desde la perspectiva de la filosofía de la historia. El autor identificaría en Lacunza una visión escatológica de la historia, proveniente del milenarismo medieval. Su estudio de La Venida del Mesías en gloria y majestad se centra en la idea de la historia y su imaginario, y con este fin analiza las llamadas "figuras" de origen bíblico (como por ejemplo, el Anticristo) que aparecen en el texto del jesuita. Rojas presenta extractos de la obra del fallecido en Imola, los que va analizando someramente, y que apoyarían su tesis principal, en la cual $L a$ Venida del Mesías en gloria y majestad estaría concebida como una forma 
de criticar a las autoridades de la Iglesia y de la política de aquella época. En este libro también se incluye un estudio sobre José Ignacio Molina (Historia Natural e Historia Civil) y su labor naturalista, mostrándolos como las dos vertientes de un pensamiento crítico ilustrado.

Parra, Fredy. "Manuel Lacunza: su obra y su visión de la historia”. La Revista Católica $\mathrm{n}^{\mathrm{o}} 1.133$ (2002); pp. 29-38.

En este artículo, el autor analiza la obra La Venida del Mesías en gloria $y$ majestad, para encontrar la visión de la historia que proponía Lacunza, en concordancia con su pensamiento mesiánico-milenarista. Según Parra, Lacunza enfoca su interés en el fenómeno escatológico, proponiendo una visión futurista y anti-progreso de la historia.

Millar Carvacho, René. "Recepción de Lacunza en Chile". AHLG n XI, 2002, pp. 129-140.

Este trabajo tiene como intención mostrar la recepción que ha tenido la obra de Lacunza en Chile. Con este fin, divide la repercusión de La Venida del Mesías en gloria y majestad en cuatro momentos: en la época cercana a la aparición del libro, destacando las pasiones encontradas que suscitó la obra del jesuita a nivel teológico. El segundo momento, Millar lo sitúa entre fines del XIX y principios del XX, destacando la polémica entre Miguel R. Urzúa, Olegario Lazo, y Emilio Vaisse. El tercer momento, desde 1930, el autor pone énfasis en la influencia de Lacunza en el grupo de jóvenes católicos de aquella época. En el cuarto momento desde 1960 en adelante, se destaca el interés del mundo académico, sobre todo de Mario Góngora.

Como conclusión, Millar Carvacho, dice que a nivel popular la obra de Lacunza no ha tenido mucha repercusión, sino que se ha movido en ambientes intelectuales y eclesiásticos.

Zeballo, Ana de. "La Venida del Mesías de Manuel Lacunza. Primeras ediciones y críticas". Anuario de historia de la Iglesia, ISSN 1133-0104, n 11, 2002, pp. 115-128.

La autora, en este estudio, realiza una breve biografía de Lacunza, para después revisar las primeras ediciones del texto La Venida del Mesías en gloria y majestad, y las posteriores críticas y condenas de los contemporáneos del jesuita chileno. Además en este texto se hace una recopilación de las 
opiniones favorables sobre la obra de Lacunza, terminando con un segmento acerca del interés actual que ha provocado los postulados del chileno muerto en Imola.

Parra, Fredy. "Historia y escatología en Manuel Lacunza: La temporalidad a través del milenarismo lacunziano". Teología y Vida. Vol. 44, № 2-3 (2003); pp. 163-187.

Este trabajo analiza los componentes del sistema propuesto por Manuel Lacunza en su obra La Venida del Mesías en gloria y majestad. Lo central de la labor del autor está destinado a clarificar la concepción del tiempo que se desprende del texto del jesuita: nociones como tiempo, tiempos, eones, eternidad, parusía, kairós, resultan para el estudioso fundamentales para comprender las ideas milenarista y mesiánica del chileno.

Unzueta Castillo, Luis. "Los temas en las cartas del padre Manuel Lacunza”. En: www.uchile.cl/facultades/filosofía/cestculturales/lacunza/html

En este trabajo, Unzueta se dedica a estudiar las cartas escritas por Manuel Lacunza a sus familiares en Chile. Para este estudio, el autor introduce el marco teórico de la carta, para después identificar los temas que aparecen en las cartas, como el exilio, la soledad, y la nostalgia.

Seperiza Pasquali, Iván. "Lacunza el milenarista”. Ciberscrito n 65, Mundo Mejor: www.isp.co.cl/lacunza.htm

Trabajo en tono de homenaje que se le hace en el aniversario $\mathrm{n}^{\mathrm{o}} 200 \mathrm{de} \mathrm{su}$ muerte. Incluye fragmentos escogidos de La Venida, sus cartas y además contiene extractos de repuestas de Miguel Rafael Urzúa en contra de opositores y críticos de la obra de Lacunza. Los párrafos escogidos se refieren a la interpretación bíblica, la defensa de los milenarios, el Anticristo, entre otros. A partir de esta selección, Seperiza propone un Anti-apocalipsis, una era futura de cambio, donde se mezclan la ciencia y la religión.

Sergio Olivares. "Manuel Lacunza: La conexión adventista". Diálogo Universitario, revista internacional de fe, pensamiento y acción. http://dialogue. adventist.org/articles/06_1_olivares_s.htm

En este artículo, Olivares presenta una pequeña reseña bibliográfica de Lacunza, una síntesis de sus doctrinas y sus repercusiones. Todo esto con el 
propósito de mostrar las diferencias con los postulados de la Iglesia adventista. Por ejemplo, ambos coinciden en tomar a la Biblia como la fuente principal de estudio, y la venida del Mesías y su reino milenario. Diferencias fundamentales que alejan a la Iglesia adventista del jesuita son la concepción del Anticristo, el rechazo al rol protagónico del pueblo judío, entre otros. 